\title{
Elevated Hu-Antigen Receptor (HuR) Expression is Associated with Tumor Aggressiveness and Poor Prognosis but not with COX-2 Expression in Invasive Breast Carcinoma Patients
}

\author{
Constantinos Giaginis ${ }^{1,2}$ • Anastasia Sampani ${ }^{1}$ - Iolly Kotta-Loizou ${ }^{1}$. \\ Ioanna Giannopoulou ${ }^{1} \cdot$ Eugene Danas $^{1} \cdot$ Ekaterini Politi $^{1} \cdot$ Gerasimos Tsourouflis $^{3}$. \\ Gregorios Kouraklis $^{3}$ • Efstratios Patsouris ${ }^{1}$ • Antonios Keramopoulos ${ }^{4}$ • \\ Lydia Nakopoulou ${ }^{1} \cdot$ Stamatios Theocharis $^{1,5}$
}

Received: 24 April 2016/Accepted: 9 August 2017

(C) Arányi Lajos Foundation 2017

\begin{abstract}
Hu-antigen R (HuR), a RNA-binding protein, is considered to play a crucial role in tumor development and progression by stabilizing or regulating a group of cellular mRNAs of cancer-related genes, such as cyclooxygenase-2 (COX-2). The present study aimed to evaluate the clinical significance of $\mathrm{HuR}$ and COX-2 expression in invasive breast carcinoma. HuR and COX-2 protein expression was assessed immunohistochemically on paraffin-embedded breast cancer tissue sections obtained from 121 patients and was statistically analyzed with clinicopathological parameters, estrogen receptor (ER), progesterone receptor (PR) and human epidermal growth factor receptor 2 (HER2), as well as with tumor cells' proliferative capacity and overall and disease-free patients' survival. High HuR expression was positively associated with larger tumor size and advanced disease stage $(p=0.0234$ and $p=0.0361$, respectively), being more frequently observed in ER negative cases $(p=0.0208)$. High COX-2 expression was negatively associated with histological $(p<0.0001)$ and
\end{abstract}

Stamatios Theocharis

stamtheo@med.uoa.gr; statheocharis@yahoo.com

1 First Department of Pathology, Medical School, University of Athens, Athens, Greece

2 Department of Food Science and Nutrition, University of the Aegean, Myrina, Lemnos, Greece

3 Second Department of Propedeutic Surgery, Medical School, University of Athens, Athens, Greece

4 Department of Clinical Therapeutics, Alexandra General Hospital, Medical School, University of Athens, Athens, Greece

5 First Department of Pathology, Medical School, National and Kapodistrian University of Athens, 75 M. Asias str., Goudi, GR11527 Athens, Greece nuclear ( $p=0.0033$ ) grade and tumor cells' proliferative rate $(p=0.0015)$, being more frequently observed in luminal-A compared to other molecular subtypes $(p=0.0221)$. High HuR expression was associated with poor overall and disease-free patients' survival at both univariate (log-rank test, $p=0.0092$ and $p=0.0004$, respectively) and multivariate (Cox-regression analysis, $p=0.0223$ and $p=0.0004$, respectively) level. On the other hand, high COX-2 expression was associated with favorable overall and disease-free patients' survival merely at univariate level (log-rank test, $p=0.0389$ and $p=0.0154$, respectively). HuR expression was not associated with COX-2 expression (Spearman $R=0.1489$, $p=0.1032$ ). The present data support evidence that HuR is associated with tumor aggressiveness and poor prognosis in breast carcinoma, reinforcing its potential as promising therapeutic target in this type of neoplasia.

Keywords Hu-antigen R · COX-2 · Breast cancer . Immunohistochemistry $\cdot$ Clinicopathological parameters . Patients' prognosis

\section{Introduction}

Hu-antigen R (HuR) or ELAV (embryonic lethal, abnormal vision, Drosophila)-like protein 1 (ELAVL1) belongs to the $\mathrm{Hu} / \mathrm{ELAV}$ family and is an ubiquitously expressed RNAbinding post-transcriptional regulator [1]. HuR contains three highly conserved RNA binding domains that belong to the RNA recognition motif (RRM) superfamily [2]; RRM-1 and -2 bind to AU-rich elements (ARE), while RRM-3 binds to the mRNA poly(A) tail. A U-rich sequence approximately 17-20 nucleotides long, usually located within the $3^{\prime}$ untranslated 
region (UTR) of the target mRNAs, has been identified as the RNA motif recognised by HuR [3]. HuR binds to this motif and regulates the stability, translation and nucleocytoplasmic translocation of target mRNAs. More specifically, HuR binding may stabilize the mRNA, indirectly increasing protein production [4], while its direct effect on translation efficiency can be either positive or negative $[5,6]$. Moreover, mRNA polyadenylation, a procedure taking place in the nucleus, can also be modulated by HuR [7]. Additionally, HuR can be transported from the nucleus, where is most abundantly localized, to the cytoplasm, along with the bound mRNA [8] and this change in subcellular localization appears to be linked to the regulation of HuR function [9]. Notably, HuR can stabilize the mRNA of cyclooxygenase-2 (COX-2), an enzyme that catalyzes the synthesis of prostaglandins, and is associated with the promotion of carcinogenesis and tumor cell resistance to apoptosis $[10,11]$.

Breast cancer represents the most common malignancy and cause of cancer-related death, amongst women. Mammary tumors present highly complexity and heterogeneity, while global understanding of the underlined molecular mechanisms governing their origin and progression is still lucking [12]. Molecular imaging has been considered to exert a promising role in complementing and overcoming some of the limitations of traditional biomarkers by providing the ability to perform noninvasive, repeatable whole-body assessments [13]. Estrogen receptor (ER), progesterone receptor (PR) and human epidermal growth factor receptor 2 (HER2) define prognosis and identify tumors for targeted therapy, and remain the sole established single-molecule biomarkers defining the minimum breast cancer pathology data set [14]. ER-targeted endocrine therapies are effective for the treatment of patients with ER-positive breast tumors and tamoxifen is the most widely used endocrine anti-estrogen treatment. Interestingly, a number of studies has implicated HuR in ER and HER2 expression regulation and tamoxifen resistance, suggesting that HuR may play a crucial role in breast cancer development and possibly treatment, as recently reviewed by our group [15].

The most comprehensive studies so far have supported substantial evidence that HuR is implicated in several pathological conditions, such as atherosclerosis [16], tissue ischemia [17], pathologic inflammation [6] and neoplasia $[15,18,19]$. Notably, HuR has been suggested to participate in malignant transformation process by controlling cancerrelevant genes related with angiogenesis, differentiation, cell cycle, apoptosis, inflammatory response and cell signaling [15, 18-21]. Moreover, HuR appears to exert a critical role in tumor formation, growth and metastasis by binding to mRNA encoding proteins and by affecting their expression via mRNA stabilization and/or altered translation
[15, 20, 21]. Enhanced HuR expression and cytoplasmic localization have been associated with malignant phenotype and poor patients' prognosis in several human malignancies [18, 22]. Interestingly, several studies have further suggested that HuR is implicated in malignant transformation of the breast, however, contradictory data have currently been reported regarding its clinical and prognostic impact in this type of neoplasia [23-31]. In view of the above considerations, the present study is aimed to evaluate the immunohistochemical expression of HuR and COX-2 in invasive breast carcinoma in association with multiple clinicopathological characteristics, tumor cells' proliferative capacity and ER, PR and HER2 expression, as well as overall and disease-free patients' survival.

\section{Patients and Methods}

\section{Patients}

One hundred twenty-one invasive breast carcinoma specimens obtained from an equal number of patients who underwent surgical resection due to breast cancer were included. The patients' age ranged from 33 to 85 years (mean 57 years). None of the patients had pre-operatively received radiation or chemotherapy. The institutional ethical committee of the Medical School of the University of Athens approved this study. Informed consent was signed by all patients in order to use for research purposes their biological samples and clinical data [32].

Haematoxylin and eosin staining was performed for routine histological examination. All cases were classified in accordance with World Health Organization criteria [32] and were classified as ductal or lobular. Nuclear grading was based on nuclear pleomorphism. Staging at the time of diagnosis was based on the TNM system [33]. The combined histological grade (1, 2 or 3$)$ of infiltrating ductal and lobular breast carcinomas was obtained according to the modified Scarff-BloomRichardson histological system and the guidelines suggested by Nottingham City Hospital pathologists [34]. The clinicopathological characteristics of the series are shown in Table 1.

The patients were followed up for a time interval of 8 up to 210 months with a mean survival time of $81.44 \pm 10.34$ months. Overall survival was defined as the time interval between the date of surgery and the date of death due to breast carcinoma or the last follow-up. Disease-free survival was defined as the time interval between the date of surgery and the date of detection of recurrence or the date of last follow-up without recurrence for breast carcinoma. At the time of the last follow-up, $25(20.7 \%)$ patients had died from disease, $15(12.4 \%)$ were alive with disease and 81 
Table 1 Associations of $\mathrm{HuR}$ and COX-2 expression with clinicopathological parameters in 121 invasive breast carcinoma patients

\begin{tabular}{|c|c|c|c|c|c|c|}
\hline \multirow[t]{2}{*}{ Clinicopathological parameters } & \multicolumn{3}{|c|}{ HuR expression } & \multicolumn{3}{|c|}{ COX-2 expression } \\
\hline & Low $(\%)$ & High $(\%)$ & $p$-value & Low $(\%)$ & $\operatorname{High}(\%)$ & $p$-value \\
\hline$N=121$ & $67(55.4)$ & $54(44.6)$ & & $65(53.7)$ & $56(46.3)$ & \\
\hline Age (mean $\pm \mathrm{SD} ; \mathrm{ys})$ & & & 0.9349 & & & 0.6534 \\
\hline$\leq 57.0 \pm 12.5 \mathrm{yrs}$ & $33(27.3)$ & $27(22.3)$ & & $31(25.6)$ & $29(24.0)$ & \\
\hline$>57.0 \pm 12.5 \mathrm{yrs}$ & $34(28.1)$ & $27(22.3)$ & & $34(28.1)$ & $27(22.3)$ & \\
\hline Menopausal status & & & 0.3860 & & & 0.5818 \\
\hline Premenopausal & $21(17.4)$ & $21(17.4)$ & & $24(19.8)$ & $18(14.9)$ & \\
\hline Postmenopausal & $46(38.0)$ & $33(27.3)$ & & $41(33.9)$ & $38(36.4)$ & \\
\hline Histopathological type & & & 0.6330 & & & 0.3583 \\
\hline Ductal & $47(38.8)$ & $40(33.1)$ & & $49(40.5)$ & $38(31.4)$ & \\
\hline Lobular & $20(16.5)$ & $14(11.6)$ & & $16(13.2)$ & $18(14.9)$ & \\
\hline Histological Grade & & & 0.0921 & & & $<0.0001$ \\
\hline $1+2$ & $46(38.0)$ & $29(24.0)$ & & $27(22.3)$ & $48(39.7)$ & \\
\hline 3 & $21(17.4)$ & $25(20.7)$ & & $38(31.4)$ & $8(6.6)$ & \\
\hline Nuclear Grade & & & 0.0606 & & & 0.0033 \\
\hline 1 & $35(28.9)$ & $19(15.7)$ & & $21(17.4)$ & $33(27.3)$ & \\
\hline $2+3$ & $32(26.5)$ & $35(28.9)$ & & $44(36.4)$ & $23(19.0)$ & \\
\hline Molecular subtype & & & 0.0793 & & & 0.0221 \\
\hline Luminal-A & $24(19.8)$ & $20(16.5)$ & & $16(13.2)$ & $28(23.1)$ & \\
\hline Luminal-B & $28(23.1)$ & $12(9.9)$ & & $26(21.5)$ & $14(11.6)$ & \\
\hline HER2 & $5(4.1)$ & $7(5.8)$ & & $9(7.4)$ & $3(2.5)$ & \\
\hline Triple negative & $10(8.3)$ & $15(12.4)$ & & 14 (11.6) & $11(9.1)$ & \\
\hline Tumor size & & & 0.0234 & & & 0.0535 \\
\hline$<2 \mathrm{~cm}$ & $25(20.7)$ & $10(8.3)$ & & 14 (11.6) & $21(17.4)$ & \\
\hline$\geq 2 \mathrm{~cm}$ & $42(34.7)$ & $44(36.4)$ & & $51(42.1)$ & $35(28.9)$ & \\
\hline Lymph nodes & & & 0.4650 & & & 0.4463 \\
\hline Non infiltrated & $33(27.3)$ & $23(19.0)$ & & $28(23.1)$ & $28(23.1)$ & \\
\hline Infiltrated & $34(28.1)$ & $31(25.6)$ & & $37(30.6)$ & $28(23.1)$ & \\
\hline Histopathological stage & & & 0.0361 & & & 0.1465 \\
\hline I & $22(18.2)$ & $7(5.8)$ & & $11(9.1)$ & $18(14.9)$ & \\
\hline II & $36(29.8)$ & $36(29.8)$ & & $42(34.7)$ & $30(24.8)$ & \\
\hline III + IV & $9(7.4)$ & $11(9.1)$ & & $12(9.9)$ & $8(6.6)$ & \\
\hline ER expression & & & 0.0208 & & & 0.0892 \\
\hline Negative & $22(18.2)$ & $29(24.0)$ & & $32(26.4)$ & $19(15.7)$ & \\
\hline Positive & $45(37.2)$ & $25(20.7)$ & & $33(27.3)$ & $37(30.6)$ & \\
\hline PR expression & & & 0.2384 & & & 0.9327 \\
\hline Negative & $30(24.8)$ & $30(24.8)$ & & $32(26.4)$ & $28(23.1)$ & \\
\hline Positive & $37(30.6)$ & $24(19.8)$ & & $33(27.3)$ & $28(23.1)$ & \\
\hline HER2 expression & & & 0.7574 & & & 0.1615 \\
\hline Negative & $57(47.1)$ & $47(38.8)$ & & $52(43.0)$ & $50(41.3)$ & \\
\hline Positive & $10(8.3)$ & $7(5.8)$ & & $13(10.7)$ & $6(5.0)$ & \\
\hline $\mathrm{Ki}-67$ protein statement & & & 0.8065 & & & 0.0015 \\
\hline Below median value & $32(26.5)$ & $27(22.3)$ & & $23(19.0)$ & $36(29.8)$ & \\
\hline Over median value & $35(28.9)$ & $27(22.3)$ & & $42(34.7)$ & $20(16.5)$ & \\
\hline
\end{tabular}

Statistically significant $p$-values are depicted by bold
$(66.9 \%)$ were alive and disease-free. All patients received conventional postoperative treatment depending on the extent of the disease, including adjuvant chemotherapy, radiation therapy and anti-estrogen therapy, when indicated, according to the consensus recommendations at the time [35]. 


\section{Immunohistochemistry}

Commercially available rabbit polyclonal anti-HuR (H-280, sc-20,694) and anti-COX-2 (H-62, sc-7951) IgG antibodies (Santa Cruz Biochemicals, Santa Cruz, CA, USA) were used for HuR and COX-2 immunostainings on formalin-fixed, paraffin-embedded breast tissue sections. Four $\mu \mathrm{m}$ thick tissue sections were deparaffinized, rehydrated, immersed in $3 \%$ $\mathrm{H}_{2} \mathrm{O}_{2}$ for $30 \mathrm{~min}$ and microwaved at $750 \mathrm{~W}$ in $0.01 \mathrm{M}$ citrate buffer (pH 6.0) for $15 \mathrm{~min}$ and then they left to cool down in TBS. Incubation with primary HuR and COX-2 antibodies was performed for $1 \mathrm{~h}$ at room temperature $\left(37^{\circ} \mathrm{C}\right)$, at a dilution 1:100 and 1:200, respectively. The standard twostep peroxidase conjugated polymer technique (DAKO Envision kit, DAKO, Carpinteria, CA, USA) was then performed. At a next step, immunostainings were visualized with diaminobenzidine tetrahydrochloride solution (DAB; Sigma, Saint Louis, MO, USA). Sections were counterstained with Harris' hematoxylin and mounted in Entellan (Merck, Darmstadt, Germany). Appropriate negative controls were performed by omitting the primary HuR and COX-2 antibodies and/or substituting them with an irrelevant anti-serum. Lung and thyroid cancer tissue sections with known enhanced HuR and COX-2 expression were used as positive control [19, 36]. A mouse anti-human Ki-67 antigen; IgG1k antibody (clone MIB-1, Dakopatts, Glostrup, Denmark) were used to evaluate the tumor cells' proliferative capacity $[36,37]$. The expression of ER, PR and HER2 was assessed immunohistochemically, as previously described [38].

\section{Evaluation of Immunohistochemistry}

Immunohistochemical evaluation was performed by counting at least 1000 tumor cells in each case by two independent observers blinded to the clinical data. Specimens were considered HuR and COX-2 -positive when more than 5\% of tumor cells within the section were positively stained. HuR and COX-2 immunoreactivity was scored according to the percentage of positive tumor cells as 0 : negative staining- $0-4 \%$ of tumor cells positive; $1: 5-24 \%$ of tumor cells positive; 2 : $25-49 \%$ of tumor cells positive; $3: 50-100 \%$ of tumor cells positive, and its intensity as 0 : negative staining, 1 : mild staining; 2: intermediate staining; 3 : intense staining. Finally, HuR and COX-2 expression was classified as low; if the total score was 0 or 2 and high; if the total score was $\geq 3$. In this way, we ensure that each group has a more homogeneous and sufficient number of cases in order to be comparable with the other groups $[19,36,37,39]$.

Staining for ER and PR was evaluated according to CAP/ ASCO recommendations, i.e. ER and PR assays are considered positive if there are at least $1 \%$ positive tumor nuclei in the sample in the presence of the expected reactivity of internal and external controls [40]. The fraction of HER2 positive stained cells was scored according to CAP/ASCO guidelines [41]. Ki-67 immunoreactivity was classified according to the percentage of positively stained breast cancer cells exceeded the median percentage value into two categories (below and over mean value), as previously reported [19, 36, 37, 39].

\section{Statistical Analysis}

The associations of HuR and COX-2 protein expression with clinicopathological variables, tumor cells' proliferative capacity and ER, PR and HER2 protein expression were evaluated by chi-square test. The Kaplan-Meier method was applied to construct survival curves and the log rank test was applied to compare the differences between the curves. To assess, at a multivariate level, the associations between the potential prognostic marker and overall and disease-free patients' survival, a Cox proportional-hazard regression model was developed. A $p$-value lower than 0.05 was considered as the limit of statistical significance. For all analyses SPSS for Windows Software was used (SPSS Inc., 2003, Chicago, USA).

\section{Results}

\section{Associations of HuR Expression with Clinicopathological Parameters and Patients' Survival}

HuR positivity (IHC score $>0$ ) was noted in $104(86.0 \%)$ out of 121 breast cancer cases. The intensity of HuR immunostaining was mild in $39(37.5 \%)$, moderate in $42(40.4 \%)$ and intense in $23(22.1 \%)$ out of 104 HuR-positive breast carcinoma cases. Fifty-four (44.6\%) out of the 121 examined cases presented high HuR expression (IHC score $\geq 3$ ). The subcellular pattern of HuR distribution was cytoplasmic in $102(84.3 \%)$ and both cytoplasmic and nuclear in 19 $(15.7 \%)$ out of the 121 examined cases. Normal surrounding areas adjacent to tumor were found either negative or presented mild nuclear immunostaining for HuR. Representative HuR cytoplasmic or cytoplasmic and nuclear immunostainings are depicted in Fig. 1a and b, respectively. Seventy (57.9\%) out of 121 breast carcinoma cases were ER positive. PR positivity was noted in $61(50.4 \%)$ out of 121 breast carcinoma cases, while $17(14.1 \%)$ cases were HER2 positive. Sixty-five $(53.7 \%)$ out of 121 breast carcinoma cases were classified as luminal-A, 17 (14.1\%) cases as luminal-B, $25(20.7 \%)$ case as triple negative and $14(11.6 \%)$ as HER2 phenotype.

In cross-tabulation, high HuR expression was significantly associated with larger tumor size and advanced disease stage (Table $1, p=0.0234$ and $p=0.0361$, respectively). High HuR expression was significantly more frequently observed in ER negative breast carcinoma cases (Table $1, p=0.0208$ ). Borderline associations between high HuR expression and 
Fig. 1 Representative immunostainings for $\mathbf{a}$. Cytoplasmic and nuclear HuR expression (X400) b.

Cytoplasmic HuR expression (X400) and c. Cytoplasmic COX2 expression (X400).

Streptavidin-biotin-peroxidase, DAB chromogen, Harris hematoxylin counterstain
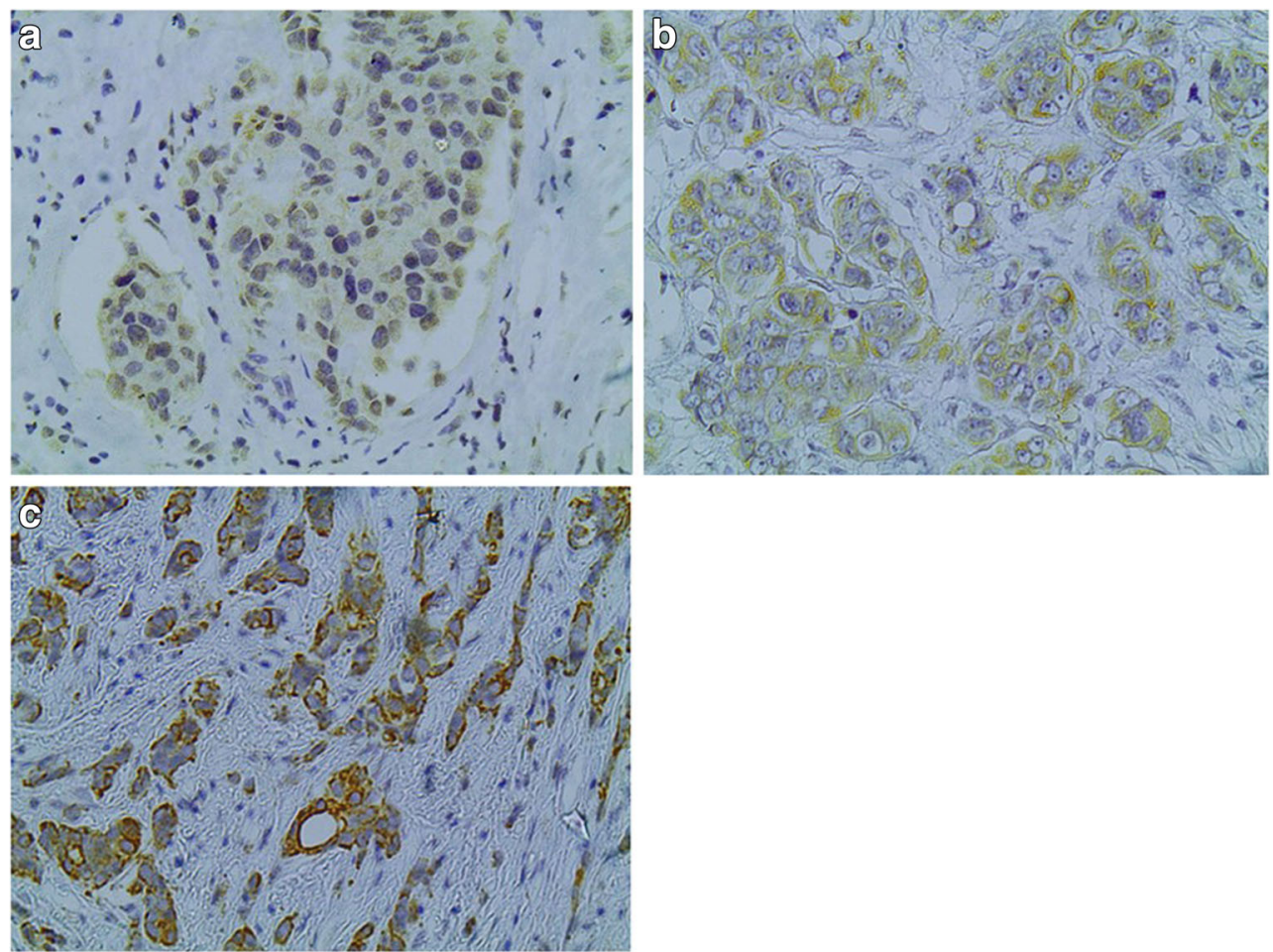

histological and nuclear grade of differentiation were recorded (Table $1, p=0.0921$ and $p=0.0606$, respectively). High HuR expression was more frequently observed in PR negative breast carcinoma cases, at a no significant level though (Table $1, p=0.2384$ ). Triple negative and HER2 subtype breast carcinoma cases presented an increased incidence of high HuR expression compared to luminal-A and luminal-B molecular subtypes (Table 1, $p=0.0793$ ). Cytoplasmic subcellular HuR distribution was significantly more frequently observed in postmenopausal breast carcinoma patients ( $p=0.0207)$, as well as in those presenting tumor infiltrated lymph nodes $(p=0.0350)$. Subcellular HuR distribution was not associated with either any of the other clinicopathological parameters examined or patients' survival (data not shown).

Kaplan-Meier survival curves indicated that breast carcinoma patients presenting high HuR expression showed significantly shorter overall survival times compared to those with low HuR expression (Fig. 2a, log-rank test, $p=0.0092$ ). In multivariate analysis, histological type and grade, tumor size, HER2 expression, Ki-67 protein statement and HuR expression were identified as independent prognostic factors of overall patients' survival (Table 2, Cox-regression analysis, $p=0.0223, p=0.0495, p=0.0316, p=0.0272, p<0.0001$ and $p=0.0184$, respectively). Kaplan-Meier survival curves indicated that breast carcinoma patients presenting high HuR expression showed significantly shorter disease-free survival times compared to those with low HuR expression (Fig. 2b, log-rank test, $p=0.0002$ ). In multivariate analysis, histological type and grade, tumor size, HER2 expression, Ki-67 protein statement and HuR expression were identified as independent prognostic factors of disease-free patients' survival (Table 3, Cox-regression analysis, $p=0.0057, p=0.0434$, $p=0.0326, p=0.0018, p=0.0001$ and $p=0.0004$, respectively).

\section{Associations of COX-2 Expression with Clinicopathological Parameters and Patients' Survival}

COX-2 positivity (IHC score $>0$ ) was noted in $93(76.9 \%$ ) out of 121 breast cancer cases. The intensity of COX-2 immunostaining was mild in $17(18.3 \%)$, moderate in $38(40.9 \%)$ and intense in 38 (40.9\%) out of 93 COX-2 positive breast carcinoma cases. Fifty-six (46.3\%) out of the 121 examined cases presented high COX-2 expression (IHC score $\geq 3$ ). The subcellular pattern of COX distribution was cytoplasmic in all the examined cases. Normal surrounding areas adjacent to tumor were found either negative or presented mild cytoplasmic immunostaining for COX-2. Representative COX-2 immunostaining is depicted in Fig. 1c.

In cross-tabulation, high COX-2 expression was negatively associated with histological and nuclear grade (Table 1, $p<0.0001$ and $p=0.0033$, respectively). Luminal-A molecular subtype breast carcinoma cases presented a significantly increased incidence of high COX-2 expression compared to luminal-B, triple negative and HER2 subtypes (Table 1, $p=0.0221)$. High COX-2 expression was marginally associated with lower tumor size (Table $1, p=0.0535$ ). High COX-2 

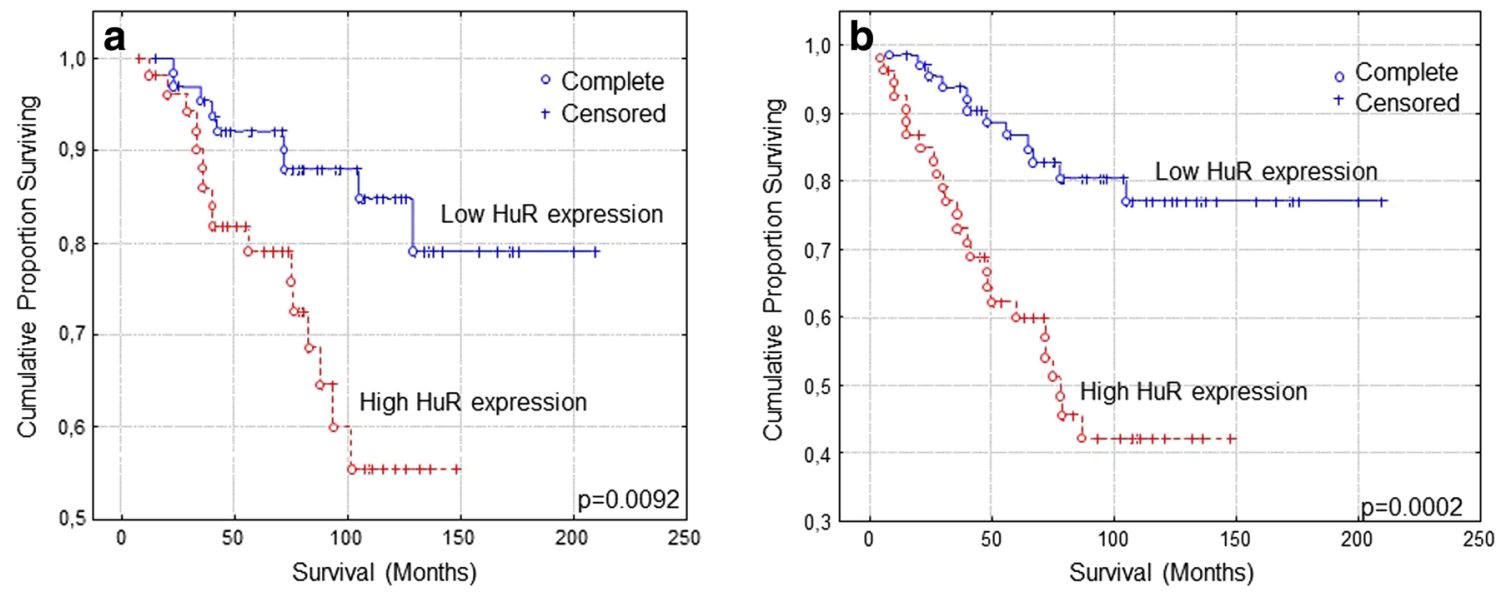

Fig. 2 Kaplan-Meier survival analysis stratified according to HuR expression in 121 breast carcinoma patients for: a. Overall patients' survival and b. Disease-free patients' survival

expression was significantly negatively associated with tumor cells' proliferative rate (Table $1, p=0.0015$ ), presenting also a trend of positive association with ER expression (Table 1, $p=0.0892$ ). Spearman rank order correlation analysis revealed that HuR expression was not associated with COX-2 expression (Spearman $R=0.1489, p=0.1032$ ). HuR subcellular distribution was not also correlated with COX-2 expression $(p=0.3689)$.

Kaplan-Meier survival curves indicated that breast carcinoma patients presenting high COX-2 expression showed significantly longer overall survival times compared to those with low COX-2 expression (Fig. 3a, log-rank test, $p=0.0389$ ). In multivariate analysis, histological type and grade, tumor size and Ki-67 protein statement but not COX2 expression were identified as independent prognostic factors of overall patients' survival (Table 4 , Cox-regression analysis, $p=0.0104, p=0.0212, p=0.0073, p=0.0008$ and $p=0.3683$, respectively). Kaplan-Meier survival curves also indicated that breast carcinoma patients presenting high COX2 expression showed significantly longer disease-free survival times compared to those with low COX-2 expression (Fig. 3b, $\log$-rank test, $p=0.0154)$. In multivariate analysis, histological type and grade, tumor size and Ki-67 protein statement but not COX-2 expression were identified as independent prognostic factors of overall patients' survival (Table 5, Coxregression analysis, $p=0.0047, p=0.0194, p=0.0022$, $p=0.0026$ and $p=0.0836$, respectively).

\section{Discussion}

A gradually increasing number of studies have currently documented that HuR overexpression and cytoplasmic localization are associated with crucial clinicopathological parameters for patients' management and prognosis in several types of human malignancy, as recently reviewed by our group [15]. Moreover, a number of clinical studies in breast cancer patients have demonstrated that elevated HuR expression was correlated with crucial clinicopathological parameters and patients' survival, indicating that high HuR expression levels may constitute an aggravating factor for tumor growth and metastasis [15]. However, as far as concern breast carcinoma, opposite data also exist, supporting the notion that low HuR expression levels are associated with tumor aggressiveness and poor prognosis [15]. Moreover, a lot of currently available data supported substantial evidence that COX-2 is associated with the promotion of carcinogenesis and tumor cell resistance to apoptosis $[10,11]$.
Table 2 Multivariate analysis for HuR expression and overall patients' survival

\begin{tabular}{lll}
\hline Clinicopathological variables & Overall survival & \\
\cline { 2 - 3 } & HR $(95 \% \mathrm{CI})$ & $p$-value \\
\hline Histological type (Ductal / Lobular) & $0.266(0.019-0.583)$ & $\mathbf{0 . 0 2 2 3}$ \\
Histological grade (I + II / III) & $0.423(0.122-1.034)$ & $\mathbf{0 . 0 4 9 5}$ \\
Tumor size (<2 cm / > 2 cm) & $9.900(4.132-18.950)$ & $\mathbf{0 . 0 3 1 6}$ \\
HER-2 expression (Negative / Positive) & $3.193(1.383-6.785)$ & $\mathbf{0 . 0 2 7 2}$ \\
Ki-67 statement (Below / Over median value) & $9.489(6.731-12.549)$ & $<\mathbf{0 . 0 0 0 1}$ \\
HuR expression (Low / High) & $2.948(1.294-4.789)$ & $\mathbf{0 . 0 1 8 4}$ \\
\hline
\end{tabular}

Statistically significant $p$-values are depicted by bold 
Table 3 Multivariate analysis for HuR expression and disease-free patients' survival

\begin{tabular}{lll}
\hline Clinicopathological variables & \multicolumn{2}{l}{ Disease-free survival } \\
\cline { 2 - 3 } & HR $(95 \% \mathrm{CI})$ & $p$-value \\
\hline Histological type (Ductal / Lobular) & $0.280(0.034-0.598)$ & $\mathbf{0 . 0 0 5 7}$ \\
Histological grade (I + II / III) & $0.489(0.138-1.143)$ & $\mathbf{0 . 0 4 3 4}$ \\
Tumor size (<2 cm / > 2 cm) & $3.359(1.726-7.420)$ & $\mathbf{0 . 0 3 2 7}$ \\
HER-2 expression (Negative / Positive) & $3.802(1.542-7.329)$ & $\mathbf{0 . 0 0 1 8}$ \\
Ki-67 statement (Below / Over median value) & $4.527(3.354-6.781)$ & $\mathbf{0 . 0 0 0 1}$ \\
HuR expression (Low / High) & $3.714(2.443-5.140)$ & $\mathbf{0 . 0 0 0 4}$ \\
\hline
\end{tabular}

Statistically significant $p$-values are depicted by bold
In this aspect, the present study is aimed to assess the expression levels of HuR and COX-2 in breast carcinoma in order to further clarify their clinical and prognostic impact in this type of neoplasia. According to our results, approximately half of the examined cases presented high HuR expression levels and all the examined cases presented negative or mild nuclear HuR immunostaining in non-malignant breast tissue. Moreover, it should be noted that HuR subcellular distribution was found predominately cytoplasmic in the vast majority of the examined breast carcinoma cases, which suggested that HuR may be translocated from nucleus to cytoplasm during the malignant breast transformation process.

The present study also showed that high HuR expression was associated with larger tumor size and advanced disease stage. High HuR expression was also more frequently observed in ER negative breast carcinoma cases. Borderline associations between high HuR expression and histological and nuclear grade of differentiation were also recorded, while triple negative and HER2-type breast cancer cases presented an increased incidence of high HuR expression compared to luminal-A and -B molecular subtypes. Moreover, cytoplasmic subcellular HuR distribution was more frequently observed in postmenopausal breast carcinoma patients, as well as in those presenting tumor infiltrated lymph nodes. These findings supported substantial evidence for a potential crucial role of HuR in breast malignant progression that affect patients' survival. The strong association found between high HuR expression and poor overall and disease-free patients' survival further suggested that HuR may represent a potential negative prognosticator in invasive breast carcinoma. These data also reinforce the therapeutic utility of HuR targeting in breast cancer chemoprevention, since HuR appears to be a common denominator and regulator for a number of molecular pathways crucial for tumor formation, growth and metastasis, being implicated in chemoresistance mechanisms to therapeutic drugs, such as tamoxifen, as well as being associated with important potential therapeutic targets, such as cyclin D1, CDK1, CDK7, MPP-13 and YES1 [15].

In addition, our results are in accordance to several previously published studies [23-31]. In fact, a clinical study conducted on 97 ductal breast carcinoma patients revealed significant associations between elevated total HuR expression (calculated from the combination of staining intensity and extent of positivity in tumor cells) and advanced tumor histological grade and HER2-negative status [23]. Furthermore, cytoplasmic HuR expression pattern (calculated from the combination of staining intensity and extent of positivity in tumor cells) was positively associated with histological grade in 208
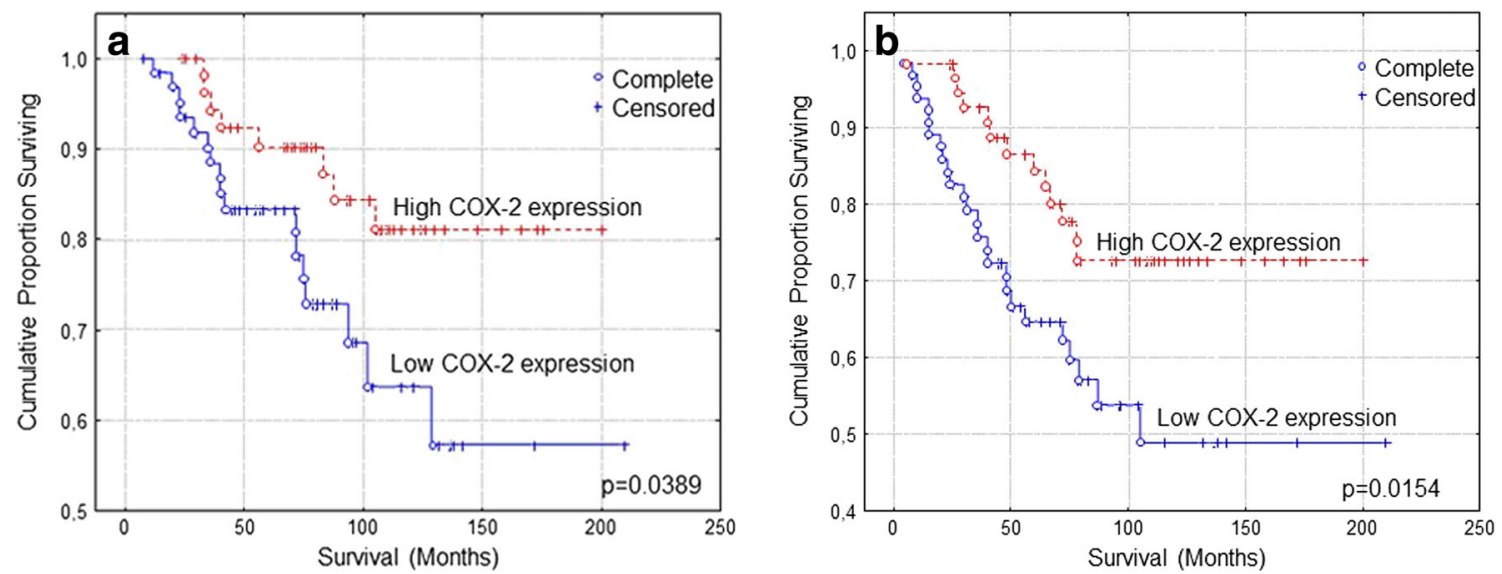

Fig. 3 Kaplan-Meier survival analysis stratified according to COX-2 expression in 121 breast carcinoma patients for: a. Overall patients' survival and b. Disease-free patients' survival 
Table 4 Multivariate analysis for COX-2 expression and overall patients' survival

\begin{tabular}{lll}
\hline Clinicopathological variables & \multicolumn{1}{l}{ Overall survival } & \\
\cline { 2 - 3 } & HR $(95 \%$ CI $)$ & $p$-value \\
\hline Histological type (Ductal / Lobular) & $0.221(0.021-0.597)$ & $\mathbf{0 . 0 1 0 4}$ \\
Histological grade (I + II / III) & $0.344(0.101-0.934)$ & $\mathbf{0 . 0 2 1 2}$ \\
Tumor size (<2 cm / > 2 cm) & $16.568(7.152-24.302)$ & $\mathbf{0 . 0 0 7 3}$ \\
HER-2 expression (Negative / Positive) & $1.980(0.583-6.673)$ & 0.1610 \\
Ki-67 statement (Below / Over median value) & $6.843(3.451-12.769)$ & $\mathbf{0 . 0 0 0 8}$ \\
COX-2 expression (Low / High) & $0.663(0.098-3.559)$ & 0.3683 \\
\hline
\end{tabular}

Statistically significant $p$-values are depicted by bold invasive breast carcinoma patients [24]. Enhanced cytoplasmic HuR staining intensity was correlated with advanced patients' age and tumor histological grade in 82 breast carcinoma patients [26], with increased tumor grade and larger tumor size in another three studies conducted on 133, 208 and 139 invasive breast carcinoma patients, respectively [24, 27, 28], as well as with increased histological grade and ductal tumor type in 525 familial non-BRCA1/2 cases [29], and in 76 invasive breast carcinoma patients receiving paclitaxel and anthracycline-based neoadjuvant chemotherapy [25]. In accordance with the present findings, enhanced $\mathrm{HuR}$ staining intensity was correlated with ER-negative status in familial non-BRCA1/2 cases [29] and in invasive carcinoma patients receiving paclitaxel and anthracycline-based neoadjuvant chemotherapy [27]. Moreover, enhanced HuR staining intensity was correlated with PR-negative status in ductal in situ breast carcinoma [25], in familial non-BRCA1/2 cases [29], and in invasive breast carcinoma patients receiving paclitaxel and anthracycline-based neoadjuvant chemotherapy [27]. In contrast, elevated cytoplasmic HuR staining intensity was associated with PR-, ER- and HER2-positive status in a more recent study conducted by Zhu et al. [26].

Regarding the role of HuR as a prognosticator in breast cancer patients and in accordance with our findings, enhanced cytoplasmic HuR protein expression was identified as an independent prognostic factor for shorter overall and/or diseasefree survival rate in ductal invasive breast carcinoma patients $[26,28]$, as also in invasive breast carcinoma patients receiving paclitaxel and anthracycline-based neoadjuvant chemotherapy [27], and in familial non-BRCA1/2 patients [29]. In contrast, other studies documented that low HuR mRNA expression was associated with poor prognosis and increased risk of disease recurrence in ductal invasive breast carcinoma patients $[30,31]$. To this point, it should be noted that the first four studies that are in accordance with our findings applied an immunohistochemical technique to quantify HuR expression at protein level, whereas the last two studies that found an opposite association between HuR expression and patients' prognosis quantified HuR expression at mRNA level by the use of reversed transcription polymerase chain reaction technique. In this aspect, the above controversy may be ascribed to potential post-transcriptional modifications of $\mathrm{HuR}$ protein that may affect its clinical and prognostic impact. Different antibodies used may also be responsible for this controversy.

The present study also revealed that high COX-2 expression was negatively associated with histological and nuclear grade, and tumor cells' proliferative rate, being more frequently observed in luminal-A compared to other molecular subtypes. High COX-2 expression was also associated with favorable patients' prognosis. These findings are in accordance with previous data [42]. Moreover, COX-2 expression was inversely associated with tumor size, disease stage and HER-2 expression and positively with ER expression, at a non-significant level though. These findings supported substantial evidence for a potential crucial role of COX-2 in breast malignant progression that affect patients' survival. Denkert
Table 5 Multivariate analysis for COX-2 expression and diseasefree patients' survival

\begin{tabular}{lll}
\hline Clinicopathological variables & \multicolumn{2}{l}{ Disease-free survival } \\
\cline { 2 - 3 } & HR $(95 \%$ CI & $p$-value \\
\hline Histological type (Ductal / Lobular) & $0.267(0.032-0.531)$ & $\mathbf{0 . 0 0 4 7}$ \\
Histological grade (I + II / III) & $0.423(0.122-1.018)$ & $\mathbf{0 . 0 1 9 4}$ \\
Tumor size (<2 cm / > 2 cm) & $5.427(2.228-8.950)$ & $\mathbf{0 . 0 0 2 2}$ \\
HER-2 expression (Negative / Positive) & $2.140(0.598-5.229)$ & 0.0522 \\
Ki-67 statement (Below / Over median value) & $3.118(1.564-5.891)$ & $\mathbf{0 . 0 0 2 6}$ \\
COX-2 expression (Low / High) & $0.536(0.098-2.320)$ & 0.0836 \\
\hline
\end{tabular}

Statistically significant $p$-values are depicted by bold 
et al. also reported that elevated HuR expression was associated with increased COX-2 expression in human breast carcinoma [24]. In contrast, we did not find any association between HuR and COX-2 expression in our cohort study, while not any other existing study has reported such an association as far as concern breast carcinoma.

\section{Conclusions}

The present study supported clinical evidence that elevated HuR expression is associated with advanced tumor aggressiveness and poor prognosis in patients with invasive breast carcinoma. Moreover, HuR translocation from nucleus to cytoplasm was observed, which may suggest that this is a potential event during malignant breast transformation process. On the other hand, COX-2 expression was associated with less tumor aggressiveness and favorable prognosis in invasive breast carcinoma patients. Additional research conducted on larger cohorts and on each molecular subtype separately should be performed in order to explore the exact molecular pathways in which HuR and COX-2 are implicated, as well as to evaluate their role in drug resistance.

\section{Compliance with Ethical Standards}

Conflict of Interest All authors verify that they have not accepted any funding or support from an organization that may in any way gain or lose financially from the results of the present study. All authors verify that they have not been employed by an organization that may in any way gain or lose financially from the results of the present study. None authors have any other conflicting interest.

\section{References}

1. Ma WJ, Cheng S, Campbell C, Wright A, Furneaux H (1996) Cloning and characterization of HuR, a ubiquitously expressed Elav-like protein. J Biol Chem 271:8144-81451

2. Burd CG, Dreyfuss G (1994) Conserved structures and diversity of functions of RNA-binding proteins. Science 265:615-621

3. Lopez de Silanes I, Zhan M, Lal A, Yang X, Gorospe M (2004) Identification of a target RNA motif for RNA-binding protein HuR. Proc Natl Acad Sci U S A 101:2987-2992

4. Brennan CM, Steitz JA (2001) HuR and mRNA stability. Cell Mol Life Sci 58:266-277

5. Kullmann M, Gopfert U, Siewe B, Hengst L (2002) ELAV/Hu proteins inhibit p27 translation via an IRES element in the p27 5'UTR. Genes Dev 16:3087-3099

6. Mazan-Mamczarz K, Galban S, Lopez de Silanes I et al (2003) RNA-binding protein HuR enhances p53 translation in response to ultraviolet light irradiation. Proc Natl Acad Sci U S A 100: 8354-8359

7. Zhu H, Zhou HL, Hasman RA, Lou H (2007) Hu proteins regulate polyadenylation by blocking sites containing U-rich sequences. J Biol Chem 282:2203-2210

8. Fan XC, Steitz JA (1998) HNS, a nuclear-cytoplasmic shuttling sequence in HuR. Proc Natl Acad Sci U S A 95:15293-11528
9. Keene JD (1999) Why is Hu where? Shuttling of early-responsegene messenger RNA subsets. Proc Natl Acad Sci U S A 96:5-7

10. Khan Z, Khan N, Tiwari RP, Sah NK, Prasad GB, Bisen PS (2011) Biology of COX-2: an application in cancer therapeutics. Curr Drug Targets 12:1082-1093

11. Ghosh N, Chaki R, Mandal V, Mandal SC (2010) COX-2 as a target for cancer chemotherapy. Pharmacol Rep 62:233-244

12. Skibinski A, Kuperwasser C (2015) The origin of breast tumor heterogeneity. Oncogene 34:5309-5316

13. Ulaner GA, Riedl CC, Dickler MN, Jhaveri K, Pandit-Taskar N, Weber W (2016) Molecular imaging of biomarkers in breast cancer. J Nucl Med 57:53S-59S

14. Kos Z, Dabbs DJ (2016) Biomarker assessment and molecular testing for prognostication in breast cancer. Histopathology 68:70-85

15. Kotta-Loizou I, Giaginis C, Theocharis S (2014) Clinical significance of HuR expression in human malignancy. Med Oncol 31:161

16. Rhee WJ, Ni CW, Zheng Z, Chang K, Jo H, Bao G (2010) HuR regulates the expression of stress-sensitive genes and mediates inflammatory response in human umbilical vein endothelial cells. Proc Natl Acad Sci U S A 107:6858-6863

17. Zhang J, Modi Y, Yarovinsky T et al (2012) Macrophage beta2 integrin-mediated, HuR-dependent stabilization of angiogenic factor-encoding mRNAs in inflammatory angiogenesis. Am J Pathol 180:1751-1760

18. Lopez de Silanes I, Lal A, Gorospe M (2005) HuR: posttranscriptional paths to malignancy. RNA Biol 2:11-13

19. Giaginis C, Alexandrou P, Tsoukalas N et al (2015) Hu-antigen receptor $(\mathrm{HuR})$ and cyclooxygenase-2 (COX-2) expression in human non-small cell lung carcinoma: associations with clinicopathological parameters, tumor proliferative capacity and patients' survival. Tumour Biol 36:315-327

20. Abdelmohsen K, Srikantan S, Kuwano Y, Gorospe M (2008) miR519 reduces cell proliferation by lowering RNA-binding protein HuR levels. Proc Natl Acad Sci U S A 105:20297-20302

21. Abdelmohsen K, Kim MM, Srikantan S et al (2010) miR-519 suppresses tumor growth by reducing HuR levels. Cell Cycle 9:1354 1359

22. Srikantan S, Gorospe M (2012) HuR function in disease. Front Biosci (Landmark Ed) 17:189-205

23. Saunus JM, French JD, Edwards SL et al (2008) Posttranscriptional regulation of the breast cancer susceptibility gene BRCA1 by the RNA binding protein HuR. Cancer Res 68:9469-9478

24. Denkert C, Weichert W, Winzer KJ et al (2004) Expression of the ELAV-like protein HuR is associated with higher tumor grade and increased cyclooxygenase-2 expression in human breast carcinoma. Clin Cancer Res 10:5580-5586

25. Heinonen M, Hemmes A, Salmenkivi K et al (2011) Role of RNA binding protein $\mathrm{HuR}$ in ductal carcinoma in situ of the breast. J Pathol 224:529-539

26. Zhu Z, Wang B, Bi J et al (2013) Cytoplasmic HuR expression correlates with P-gp, HER-2 positivity, and poor outcome in breast cancer. Tumour Biol 34:2299-2308

27. Wang J, Li D, Wang B, Wu Y (2013) Predictive and prognostic significance of cytoplasmic expression of ELAV-like protein HuR in invasive breast cancer treated with neoadjuvant chemotherapy. Breast Cancer Res Treat 141:213-224

28. Heinonen M, Bono P, Narko K et al (2005) Cytoplasmic HuR expression is a prognostic factor in invasive ductal breast carcinoma. Cancer Res 65:2157-2161

29. Heinonen M, Fagerholm R, Aaltonen K et al (2007) Prognostic role of HuR in hereditary breast cancer. Clin Cancer Res 13:6959-6963

30. Yuan Z, Sanders AJ, Ye L, Wang Y, Jiang WG (2011) Prognostic value of the human antigen $\mathrm{R}(\mathrm{HuR})$ in human breast cancer: high level predicts a favourable prognosis. Anticancer Res 31:303-310

31. Ortega AD, Sala S, Espinosa E, Gonzalez-Baron M, Cuezva JM (2008) HuR and the bioenergetic signature of breast cancer: a low 
tumor expression of the RNA-binding protein predicts a higher risk of disease recurrence. Carcinogenesis 29:2053-2061

32. Tavassoli FA, Devilee P (eds) (2003) Pathology and genetics tumors of the breast and female genital organs. IARC WHO classification of tumors, no. 4. WHO Press, Lyon, pp 25-27

33. Hermanek P, Sabin H (eds) (1992) TNM classification of international union against cancer. In TNM atlas, 3rd rev, 4th edn. Springer-Verlag, Berlin, pp 15-25

34. Robins P, Pinder S, de Klerk N (1995) Histological grading of breast carcinomas: a study of interobserver agreement. Hum Pathol 6:873-879

35. Mylona E, Vamvakaris I, Giannopoulou I et al (2013) An immunohistochemical evaluation of the proteins Wnt1 and glycogen synthase kinase (GSK)-3 $\beta$ in invasive breast carcinomas. Histopathology 62:899-907

36. Michailidi C, Giaginis C, Stolakis V et al (2010) Evaluation of FAK and Src expression in human benign and malignant thyroid lesions. Pathol Oncol Res 16:497-507

37. Giaginis C, Alexandrou P, Delladetsima I, Giannopoulou I, Patsouris E, Theocharis S (2014) Clinical significance of histone deacetylase (HDAC)-1, HDAC-2, HDAC-4, and HDAC-6 expression in human malignant and benign thyroid lesions. Tumour Biol 35:61-71

38. Mylona E, Melissaris S, Nomikos A et al (2014) Effect of BRCA1 immunohistochemical localizations on prognosis of patients with sporadic breast carcinomas. Pathol Res Pract 210:533-540

39. Giaginis C, Alexandrou P, Delladetsima I et al (2016) Clinical significance of $\mathrm{Hu}$-antigen receptor $(\mathrm{HuR})$ and cyclooxygenase- 2 (COX-2) expression in human malignant and benign thyroid lesions. Pathol Oncol Res 22:189-196

40. Hammond MEH, Hayes DF, Dowsett M et al (2010) American Society of Clinical Oncology/College of American Pathologists guideline recommendations for immunohistochemical testing of estrogen and progesterone receptors in breast cancer. J Clin Oncol 28:2786-2795

41. Wolff AC, Hammond ME, Hicks DG et al (2013) Recommendations for human epidermal growth factor receptor 2 testing in breast cancer: American Society of Clinical Oncology/College of American Pathologists clinical practice guideline update. J Clin Oncol 31: 3997-3401

42. Nakopoulou L, Mylona E, Papadaki I et al (2005) Overexpression of cyclooxygenase-2 is associated with a favorable prognostic phenotype in breast carcinoma. Pathobiology 72:241-249 